\title{
Population structure of the blood clam (Tegillarca granosa) in China based on microsatellite markers
}

\author{
Y.J. Wang ${ }^{1,2}$, Q.G. Zeng' ${ }^{1}$ and L.N. Xu ${ }^{3}$ \\ ${ }^{1}$ Key Laboratory of Applied Marine Biotechnology, Ningbo University, \\ Ministry of Education, Ningbo, China \\ ${ }^{2}$ Marine Biotechnology Laboratory, Ningbo University, Ningbo, China \\ ${ }^{3}$ College of Science \& Technology, Ningbo University, Ningbo, China \\ Corresponding author: Y.J. Wang \\ E-mail: wangyajun@yahoo.cn \\ Genet. Mol. Res. 12 (2): 892-900 (2013) \\ Received June 13, 2012 \\ Accepted January 1, 2013 \\ Published April 2, 2013 \\ DOI http://dx.doi.org/10.4238/2013.April.2.6
}

\begin{abstract}
The blood clam, Tegillarca granosa, is widely cultivated in China. We isolated 6 microsatellite loci from T. granosa and used them to investigate genetic diversity and population structure of 5 widely distributed populations of blood clam collected from eastern and southeastern China. The allele number per locus varied from 4 to 9 , and the polymorphism information content value was 0.301 to 0.830 . The mean observed and expected heterozygosities varied from 0.304 to 0.460 and 0.556 to 0.621 , respectively; the population from Yueqing had the smallest observed heterozygosity. In the neighbor-joining tree, Shandong, Fenghua and Yueqing populations clustered together, and there was geographic divergence between Shandong and Guangxi populations. Some microsatellite loci that were isolated from these mainland China samples were not found in blood clams collected from Malaysia.
\end{abstract}

Key words: Tegillarca granosa; Blood clam; Microsatellite; SSR; Population structure 
Population structure of the Chinese blood clam

\section{INTRODUCTION}

Microsatellites (simple sequence repeats; SSR) are ubiquitous in eukaryotic genomes and can be studied by PCR (Powell et al., 1996). This genetic marker is known for its co-dominant nature and high polymorphism (Scott et al., 2000). In the field of shellfish research, microsatellites have proven to be useful in genetic resource assessment, population genetic structure analysis and genetic linkage map construction. For example, Geist and Kuehn (2005) used 9 microsatellite loci to analyze genetic diversity and differentiation of the freshwater pearl mussel Margaritifera margaritifera, and Hubert and Hedgecock (2004) constructed linkage maps of the Pacific oyster Crassostrea gigas with 102 microsatellite loci. However, unlike universal primer molecular markers such as amplified fragment length polymorphism, searching for microsatellite markers is complex and cumbersome. The first step is to obtain sequence information on loci and then to design primers in the flanking regions. The traditional microsatellite isolation method aims to construct a small genomic DNA bank and to hybridize clones with radioactive labeling probes to isolate microsatellite loci (Rassmann et al., 1991). However, this method requires the screening of a large number of clones, and the positive clone ratio is relatively small. Several microsatellite-enrichment methods for improving isolation efficiency have been reported, such as membrane hybridization (Karagyozov et al., 1993) and magnetic enrichment (Carleton et al., 2002). Using these enrichment methods, microsatellite loci have been largely isolated from shellfish species. Up to June 2010, more than 2000 microsatellite sequences have been deposited in GenBank for about 51 species of Lamellibranchia, the most commercially important molluse species belonging to this class.

Tegillarca granosa (blood clam), a species of the Arcidae (Lamellibranchia), usually grows in muddy beaches of the Indian Ocean and western Pacific Ocean. As it is an economically important mollusc species, $T$. granosa has been widely cultivated in East and Southeast Asian countries. In China, this species has been intensively cultivated from northern Shandong Province to southern Guangxi Province. In some Southeast Asian countries such as Malaysia, the cultivation of this mollusc also has a long history. In recent years, offspring from artificial breeding have been released into the natural environment to compensate for the shortage of natural seed or for the purpose of stock enhancement, and few of these actions have been assessed for their influence on genetic structures and gene invasion of natural populations. For genetic resource management, it is necessary to employ highly sensitive genetic makers, such as microsatellite markers, to study the genetic variation of $T$. granosa.

Many papers on the cultivation model and artificial breeding of the blood clam have been reported, where RAPD and isozyme markers have been used in T. granosa of China (most written in Chinese). However, the use of microsatellite markers in genetic structure analysis of the blood clam has not been previously reported. In this study, microsatellite loci of $T$. granosa were isolated by the magnetic bead method and microsatellite primers were designed. We examined the genetic polymorphism of microsatellite primers and determined the heterozygosity and phylogenetic relationship of 5 geographic populations of China.

\section{MATERIAL AND METHODS}

\section{Sample collection and DNA extraction}

A total of 162 samples were used in this study, and they represented 5 populations 
(Figure 1): 32 from Hainan (HN), 36 from Guangxi (GN), 30 from Yueqing (YN), Zhejiang, 33 from Fenghua (FN), Zhejiang, and 31 from Shandong (SN). Meanwhile, primers were amplified using 18 blood clams gathered from Perak State, Malaysia (MN) at the same time. Genomic DNA was extracted from muscle tissue using a commercial DNA extraction kit (ShengGong, Shanghai, China), according to the manufacturer protocol. DNA samples were electrophoresed on a $0.8 \%$ agarose gel and concentration was adjusted to $20 \mathrm{ng} / \mu \mathrm{L}$.

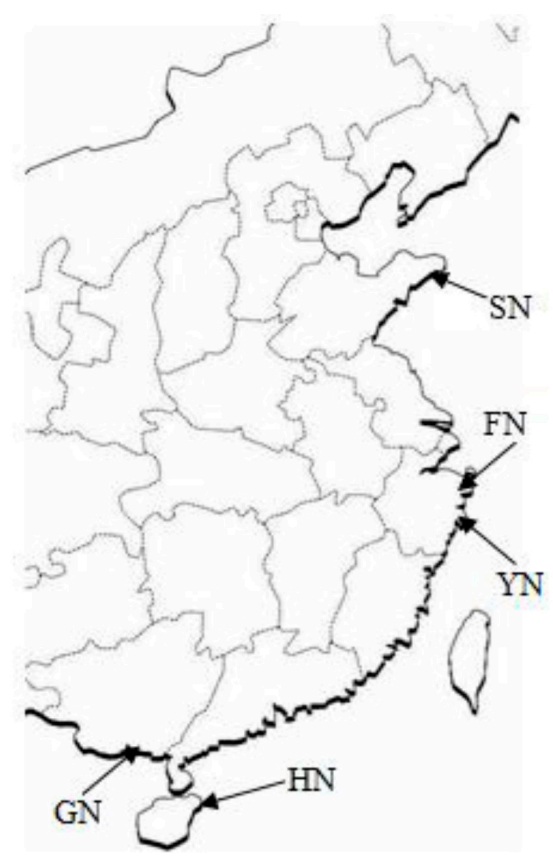

Figure 1. Location of blood clam populations from China. $\mathrm{SN}=$ Shandong; $\mathrm{FN}=$ Fenghua; $\mathrm{YN}=$ Yueqing; $\mathrm{HN}=$ Hainan; $\mathrm{GN}=$ Guangxi.

\section{Microsatellite locus isolation by magnetic bead method}

Microsatellite loci were isolated by the magnetic bead-enrichment method described by Fischer and Bachmann (1998) and Hamilton et al. (1999), with some modification. The Sau3A I adaptor-ligated fragments were hybridized with 5'-biotinylated $(\mathrm{GT})_{15},(\mathrm{CAG})_{8}$, $(\mathrm{GAA})_{8}$, and (AAAG) ${ }_{6}$ oligonucleotide probes. The hybridized fragments were then captured with streptavidin-coated magnetic Dynabeads (Dynal M-280). The enriched fragments were made double stranded by PCR with SauL-A primers (Rosas et al., 2008). PCR products were purified, ligated to Pmd18-T vector (TaKaRa) and transformed into competent cells. Clones were tested by PCR with SauL-A and oligonucleotides $(\mathrm{CAG})_{8},(\mathrm{GAA})_{8}$ or $(\mathrm{AAAG})_{6}$ as the primers, and those that produced two or more bands were selected and sequenced.

\section{Primer design and microsatellite amplification}

Eleven microsatellite sequences previously isolated by the magnetic bead method 
from a DNA pool of MN and FN were chosen to design the primers. Microsatellite primer pairs were designed by the Primer Premier version 5.0 software following the criteria that the length of the primer is 18-24 bp and the expected product size is between 100 and $300 \mathrm{bp}$. These primers were screened by temperature gradient PCR to determine the suitable annealing temperature. The functional primers were then selected to carry out population amplification. PCR was performed in a $25-\mu \mathrm{L}$ reaction system consisting of the following: $1 \mathrm{X}$ PCR buffer, $2.0 \mathrm{mM} \mathrm{MgCl}, 0.2 \mathrm{mM}$ dNTPs, 10 pmol of each primer, $0.65 \mathrm{U}$ Taq polymerase, and $10 \mathrm{ng}$ genomic DNA. The reaction was carried out in a PCR amplifier (Eppendorf Mastercycler), and the reaction program was as follows: $5 \mathrm{~min}$ at $94^{\circ} \mathrm{C}$ for denaturation, 30 cycles of $94^{\circ} \mathrm{C}$ for $1 \mathrm{~min}, 30 \mathrm{~s}$ at annealing temperature, an extension at $72^{\circ} \mathrm{C}$ for $45 \mathrm{~s}$, and a final extension step of 7 min at $72{ }^{\circ} \mathrm{C}$. PCR products were electrophoresed on an $8 \%$ polyacrylamide gel and detected by silver staining. The size of the alleles was determined according to PUC19 DNA/ MSPI (HpaII) marker as the molecular size ladder.

\section{Data analysis}

We performed alignment analysis of microsatellite-containing sequences by the DNAMAN 6.0 software to exclude redundant positive clones, and non-redundant loci were saved in FASTA format. After microsatellite analysis, the genetic diversity of every locus was testified. Expected heterozygosity $\left(H_{\mathrm{E}}\right)$ and observed heterozygosity $\left(H_{\mathrm{O}}\right)$ were estimated using Genepop 4.0.7 (Raymond and Rousset, 1995) and confirmed by CERVUS 2.0 (Marshall et al., 1998). Significant deviation from Hardy-Weinberg equilibrium (HWE) was estimated for every locus following the Markov chain method under the parameters of dememorization number = 5000 , number of batches $=20$, and iterations per batch $=2000$. For every locus, the CERVUS 2.0 program was used to calculate polymorphic information content (PIC), allele number $\left(N_{\mathrm{A}}\right)$ and total exclusionary power. The significance of the population pairwise Wright $F_{\mathrm{ST}}$ was calculated using the FSTAT software (Goudet, 1995). A phylogenetic neighbor-joining tree was constructed from $F_{\mathrm{ST}}$ in the PHYLIP software and visualized with the TREEVIEW program.

\section{RESULTS}

In this study, 228 microsatellite-containing sequences of $>10 \mathrm{bp}$, with repeat numbers not less than 5 times, were isolated from genomic DNA of T. granosa. After alignment analysis, 11 redundant sequences were excluded. Among these 217 non-redundant sequences, 186 sequences had enough flanking regions to design primers. Primarily, 11 primers were designed and tested on 5 populations of China.

Seven microsatellite loci of 11 designed primers produced targeted bands (Table 1). Among them, primer TMP35 only amplified in samples from MN, so it was excluded from the following population structure analysis. The other 6 microsatellite primers, TMP3, TMP12, TMP18, TMP22, TMP26, and TMP36, were used to study the genetic diversity and population structure of 5 blood clam populations. Among these 6 primers, TMP 18 and TMP26 also amplified in samples from $\mathrm{MN}$.

Genetic variations at the 6 microsatellite loci tested in the 5 populations are summarized in Tables 2 and 3. Among these loci, the $N_{\mathrm{A}}$ varied from 4 to 9 , with a mean allele number of 6.67. The $N_{\mathrm{A}}$ variation was positive with the microsatellite repeat times and repeat length. 
The PIC value ranged from 0.301 to 0.830 . Locus TMP12 had the highest PIC value of 0.830 compared to 0.301 for TMP36 (Table 3). For every locus, the $H_{\mathrm{E}}$ varied from 0.139 to 0.703 , while $H_{\mathrm{O}}$ varied from 0.325 to 0.851 . The total exclusionary power for the first parent was 0.902 and 0.982 for the second parent.

Table 1. Basic genetic information of microsatellite primers used in the present study.

\begin{tabular}{|c|c|c|c|c|c|c|c|c|}
\hline Locus & Repeat motif & Primer $\left(5^{\prime}-3^{\prime}\right)$ & $\mathrm{Ta}$ & $\begin{array}{l}\text { Expected } \\
\text { product } \\
\text { size (bp) }\end{array}$ & $N_{\mathrm{A}}$ & PIC & $\begin{array}{l}\text { Mean } \\
H_{\mathrm{O}} / H_{\mathrm{E}}\end{array}$ & $\begin{array}{l}\text { GenBank } \\
\text { No. }\end{array}$ \\
\hline TMP3 & $(\mathrm{GAA})_{13}$ & $\begin{array}{l}\text { F: GACAAGACGAAGTTAGCAAG } \\
\text { R: GAAACGGATAGAATAATCAAAGA }\end{array}$ & 59 & 146 & 9 & 0.818 & $0.703 / 0.842$ & EU881987 \\
\hline TMP12 & $(\mathrm{TTC})_{16}$ & $\begin{array}{l}\text { F: TTTGTCCGCTAAATCTATGTCT } \\
\text { R: TGACCAACCAAGTTTTGTAATC }\end{array}$ & 60 & 173 & 8 & 0.830 & $0.524 / 0.851$ & EU881988 \\
\hline TMP26 & $(\mathrm{AAG})_{5}$ & $\begin{array}{l}\text { F: GAAGAAGTTTGAGAATGTGG } \\
\text { R: TTTCTTTGTCTTCAGAGTCCTT }\end{array}$ & 55 & 171 & 8 & 0.458 & $0.423 / 0.497$ & EU881989 \\
\hline TMP18 & $\begin{array}{l}(\text { TTTC })_{6}\left(\text { ATC }_{4}\right. \\
\text { GTC (TTC) })_{11}\end{array}$ & $\begin{array}{l}\text { F: AGTTACAGTTGGTGTCTTTTCA } \\
\text { R: GACAGCCTCGATGTAAGTTAAA }\end{array}$ & 59 & 183 & 7 & 0.769 & $0.549 / 0.802$ & EU881990 \\
\hline TMP22 & $\begin{array}{l}(\mathrm{GAA})_{5} \mathrm{AAA} \\
(\mathrm{GAA})_{3}\end{array}$ & $\begin{array}{l}\text { F: AAAGAATCTGACAAGCCATC } \\
\text { R: CCAAAGCAATTTAACATAAC }\end{array}$ & 51 & 181 & 4 & 0.435 & $0.187 / 0.467$ & EU881991 \\
\hline $\begin{array}{l}\text { TMP36 } \\
\text { TMP35 }\end{array}$ & $\begin{array}{l}(\mathrm{CAG})_{5} \mathrm{~N} 17 \\
(\mathrm{ACA})_{4} \\
(\mathrm{AATATG})\end{array}$ & $\begin{array}{l}\text { F: ACAGGGGTCTACAAGATTCC } \\
\text { R: TGCTCATCTGTCGCTGTAAC } \\
\text { F: CTACATGAACAAGCGATACG }\end{array}$ & 61 & 164 & 4 & 0.301 & $0.139 / 0.325$ & EU881992 \\
\hline ТМР35 & $\begin{array}{l}(\text { AATATG) } \\
\left(\text { AC }_{6}\right.\end{array}$ & $\begin{array}{l}\text { F: CTACATGAACAAGCGATACG } \\
\text { R: TGTTTAATTCAGAAGGGGTG }\end{array}$ & 61 & 191 & - & - & - & EU881993 \\
\hline
\end{tabular}

$\mathrm{Ta}=$ annealing temperature; $N_{\mathrm{A}}=$ allele number; PIC $=$ polymorphic information content; $H_{\mathrm{O}}=$ observed heterozygosity; $H_{\mathrm{E}}=$ expected heterozygosity; $\mathrm{F}$ = forward primer; $\mathrm{R}=$ reverse primer.

\begin{tabular}{clllllll}
\multicolumn{7}{c}{ Table 2. Genetic variations of 6 microsatellite loci in 5 populations of blood clam. } \\
\hline Population & TMP3 & TMP12 & TMP26 & TMP18 & TMP22 & TMP36 & Mean \\
\hline GN (allele No.) & 7 & 8 & 7 & 5 & 3 & 2 & 5.3 \\
$H_{\mathrm{O}}$ & 0.857 & 0.593 & 0.355 & 0.700 & $0.231^{* *}$ & 0.028 & 0.460 \\
$H_{\mathrm{E}}$ & 0.794 & 0.740 & 0440 & 0.784 & 0.498 & 0.081 & 0.556 \\
HN (allele No.) & 7 & 8 & 5 & 7 & 4 & 3 & 5.7 \\
$H_{\mathrm{O}}$ & 0.690 & $0.531^{* *}$ & 0.563 & $0.387^{* *}$ & $0.161^{* *}$ & 0.344 & 0.446 \\
$H_{\mathrm{E}}$ & 0.817 & 0.797 & 0.495 & 0.837 & 0.454 & 0.323 & 0.621 \\
$\mathrm{SN}$ (allele No.) & 8 & 8 & 5 & 5 & 4 & 3 & 5.5 \\
$H_{\mathrm{O}}$ & 0.733 & $0.556^{* *}$ & 0.452 & $0.464^{* *}$ & $0.267^{* *}$ & 0.258 & 0.455 \\
$H_{\mathrm{E}}$ & 0.837 & 0.789 & 0.504 & 0.706 & 0.518 & 0.232 & 0.598 \\
FN (allele No.) & 5 & 7 & 7 & 6 & 4 & 3 & 5.3 \\
$H_{\mathrm{O}}$ & 0.630 & 0.688 & $0.438^{* *}$ & 0.690 & $0.121^{* *}$ & 0.121 & 0.448 \\
$H_{\mathrm{E}}$ & 0.781 & 0.848 & 0.614 & 0.777 & 0.405 & 0.117 & 0.590 \\
YN (allele No.) & 7 & 8 & 5 & 6 & 4 & 3 & 5.5 \\
$H_{\mathrm{O}}$ & $0.583^{* *}$ & $0.241^{* *}$ & 0.300 & 0.500 & $0.167 * *$ & $0.033^{* *}$ & 0.304 \\
$H_{\mathrm{E}}$ & 0.816 & 0.832 & 0.329 & 0.863 & 0.450 & 0.157 & 0.575 \\
MN (allele No.) & - & - & - & 6 & - & 1 & - \\
\hline
\end{tabular}

**Significant deviation from Hardy-Weinberg equilibrium by Markov chain procedure $(\mathrm{P}<0.01) . H_{\mathrm{O}}=$ observed heterozygosity; $H_{\mathrm{E}}=$ expected heterozygosity; $\mathrm{MN}=$ Malaysia. For other abbreviations, see legend to Figure 1.

\section{Table 3. Pairwise comparison of $F_{\mathrm{ST}}$ in 5 populations of blood clam.}

\begin{tabular}{lcccc}
\hline Population & GN & HN & SN & FN \\
\hline GN & - & - & - & - \\
HN & 0.0331 & - & - & - \\
SN & 0.0703 & 0.0290 & - & - \\
FN & 0.0477 & 0.0348 & 0.0339 & - \\
YN & 0.0498 & 0.0283 & 0.0314 & 0.0278 \\
\hline
\end{tabular}

For abbreviations, see legend to Figure 1. 
Among the 5 populations, the mean $H_{\mathrm{O}}$ and $H_{\mathrm{E}}$ varied from 0.304 to 0.460 and 0.556 to 0.621 , respectively. The YN population had the lowest $H_{\mathrm{O}}$ and $\mathrm{GN}$ had the highest value. The mean $N_{\mathrm{A}}$ was similar among these 5 populations. Deviation from HWE was assessed by the Markov chain method. Thirteen cases of deviation were detected for different loci and different populations: locus TMP3 in the YN population; TMP12 in HN, SN and YN; TMP26 in FN; TMP18 in HN and SN; TMP22 in all 5 populations; and TMP36 in YN.

The pairwise $F_{\mathrm{ST}}$ test showed that the biggest $F_{\mathrm{ST}}$ distance was between populations $\mathrm{SN}$ and GN, indicating that these two groups were more genetically divergent than other population pairs. $\mathrm{YN}$ and $\mathrm{FN}$ had the smallest genetic distance of 0.0278 , which was in accordance with the geographic distance. A neighbor-joining tree based on $F_{\mathrm{ST}}$ distance (Figure 2) showed that the FN, SN and YN populations clustered together, indicating that there was no obvious genetic differentiation between these populations. These 3 populations were slightly divergent from the $\mathrm{GN}$ and $\mathrm{HN}$ populations.

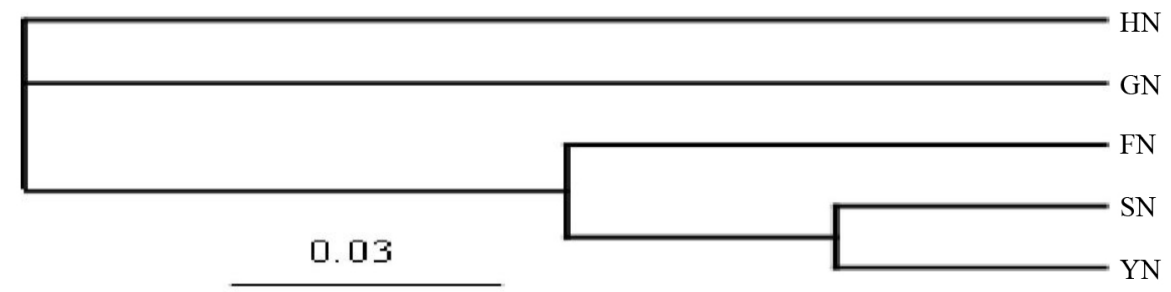

Figure 2. Neighbor-joining tree of 5 blood clam populations based on $F_{\mathrm{ST}}$ distance. For abbreviations, see legend to Figure 1.

\section{DISCUSSION}

The magnetic bead-enrichment method has been used in the isolation of microsatellites in the shellfish C. gigas (Li and Kijima, 2002) and Patinopecten yessoensis (Zhao et al., 2006). In most cases, oligonucleotide (CA/GT) was used as the probe to capture target fragments, because the $(\mathrm{CA} / \mathrm{GT})$ microsatellite motif is the most abundant sequence in genomic DNA (Brenner et al., 1993). At the same time, we also chose tri- or tetranucleotide probes to isolate microsatellite loci. In this study, the criterion for microsatellite isolation was much lower than that used by Gardner et al. (1999) (microsatellite length of more than 10 repeats), but was similar to the isolation standard of EST-SSRs from grape (Scott et al., 2000). Meanwhile, to compensate for the low discrimination of silver staining, all microsatellite primers were designed from tri- or tetranucleotide repeat motifs so as to minimize the disturbance of the stutter-band produced in most cases with dinucleotide microsatellite primer amplification.

Microsatellite DNA is a highly polymorphic genetic marker, where the polymorphism of this marker is attributed to the repeat number variation that occurred during DNA replication, damage repair and recombination (Levinson and Gutman, 1987). Previous publications proved that the longer the microsatellite repeat sequence, the higher the polymorphism of microsatellite primers (Schlötterer and Tautz, 1992; Wierdl et al., 1997; Eisen, 1999). In the present study, loci TMP22 and TMP36 had the shortest microsatellite repeats, and correspondingly, $N_{\mathrm{A}}$ and PIC values were the smallest among these 6 primers, as well as having the lowest 
$H_{\mathrm{O}}$ and $H_{\mathrm{E}}$ values. The mean $H_{\mathrm{E}}$ of the other 4 loci, estimated as being 0.497 to 0.851 , showed high genetic polymorphism.

As a relative genetic polymorphism index, mean $H_{\mathrm{O}}$ can be used to assess population genetic diversity. Among these five populations, YN had the smallest mean $H_{\mathrm{O}}$ value (0.304). Considering that the blood clam had been intensively cultivated in the Yueqing area for a long period and that artificial breeding larvae had been widely used to fill the shortage of natural breed in recent years, we believe that either samples of the $\mathrm{YN}$ population used here had experienced artificial selective pressure and some allele loss, or that the natural population in this area mingled with the artificially bred blood clam. Previous RAPD analysis showed that the wild population of Korea had higher heterozygosity than that of 4 populations of China (Li et al., 2003); these 4 populations were more or less affected by cultivation and artificial breeding seeds. Because the broodstocks for seed production have a small gene pool, the artificial offspring will cause a fluctuation in gene frequencies as well as a decrease in genetic polymorphisms in the wild (Jeong et al., 2003).

Thirteen deviations from HWE were detected in the present study and might have been due to the presence of null alleles or the consequence of small population sizes. In YN population, deviation was observed at 4 loci due to homozygote excess. Isozyme and microsatellite research has shown that heterozygote deficit may be a common observation in marine bivalve populations (Fairbrother and Beaumont, 1993; Yu and Li, 2007). However, in this case, we considered that the deviation in the YN population was caused by the introduction of a large number of artificial stock of blood clam into the wild population, which resulted in an increase in homozygotes and decrease in heterozygotes.

The $F_{\mathrm{ST}}$ value and neighbor-joining tree showed that only little genetic differentiation existed between the populations of SN, YN and FN, where the results were similar to those in previous reports. Li et al. (2003) used RAPD markers to analyze 5 populations of blood clam, and their results showed that no evident genetic differentiation existed between the Shandong and Zhejiang populations; isozyme analysis led to a similar conclusion (Wang et al., 2005). However, this situation maybe the consequence of human actions, because early in the 1960s, wild blood clam spats collected from Shandong had been cultivated in Zhejiang. Afterwards, commercial seed produced from Zhejiang, especially in the Yueqing area, were also cultivated in Shandong. In recent years, even Korean blood clam was introduced to the Yueqing area as breeding parents to undergo artificial breeding. These operations are unavoidable to minimize the genetic differentiation between different geographic populations.

Regarding the GN and $\mathrm{HN}$ populations, these two populations were clustered apart from the SN, FN and YN populations. According to Wright criteria (1978), populations GN and $\mathrm{SN}$ had undergone moderate genetic differentiation $\left(F_{\mathrm{ST}}=0.0703>0.05\right)$. Zhang et al. (2004) analyzed the genetic relationship between 4 populations of T. granosa based on 9 morphological characters, and their results showed that genetic differentiation between the Guangxi and Shandong populations was bigger than that between the Shandong and Zhejiang populations. They believed that water temperature was mainly responsible for the genetic differentiation. With respect to climate, the Shandong population is located in a temperate climate zone, where blood clams cease to grow in the winter season; the Guangxi population inhabits a subtropical area and can grow all year long. In view of geographic distance, GN and $\mathrm{HN}$ populations are far from the SN population; the geographic isolation effect is more efficient in this case. 
The total exclusionary power for first parent was 0.902 and 0.982 for the second parent. Bessert and Orti (2003) chose 7 microsatellite loci to submit to paternity analysis in the fathead minnow (Pimephales promelas), and found that the average paternity exclusion probabilities for these loci ranged from 0.102 to 0.795 , with a total paternity exclusionary power of 0.999 , and that they were suitable for paternity analysis. The total exclusionary power of these 6 microsatellite loci in this study was a little smaller than that in fathead minnow, but these loci are suitable for identifying breeding lines.

Our study showed that some microsatellite loci, which exist in the populations of China, are absent in the blood clam of Malaysia, and vice versa. Also, some primers were found to amplify on all samples from Malaysia and China. Thus, we conclude that over a long evolutionary history, significant genetic differentiation occurred between blood clams of Malaysia and China. To date, no data are available to determine if this differentiation is enough to reach the subspecies or species level.

\section{ACKNOWLEDGMENTS}

Research supported by the National High Technology Research and Development Program of China ("863" Program) (cultivation of resistant strain in clams, cockles and razor clams; \#2006AA10A410); Key Projects in the National Science \& Technology Pillar Program during the Eleventh Five-Year Plan Period from the Ministry of Science and Technology of the People's Republic of China (efficient farming and quality and safety technology development and demonstration of shellfish in East China Sea beach; \#2007BAD43B09); and K.C. Wong Magna Fund of Ningbo University. We thank Dr. Bao of Zhejiang Wanli University for comments on the manuscript.

\section{REFERENCES}

Bessert LM and Orti G (2003). Microsatellite loci for paternity analysis in the fathead minnow, Pimephales promelas (Teleostei:Cyprinidae). Mol. Ecol. Notes 3: 532-534.

Brenner S, Elgar G, Sandford R, Macrae A, et al. (1993). Characterization of the pufferfish (Fugu) genome as a compact model vertebrate genome. Nature 366: 265-268.

Carleton KL, Streelman JT, Lee BY, Garnhart N, et al. (2002). Rapid isolation of CA microsatellites from the tilapia genome. Anim. Genet. 33: 140-144.

Eisen JA (1999). Mechanistic Basis for Microsatellite Instability. In: Microsatellites: Evolution and Applications (Goldstein DB and Schlötterer C, eds.). Oxford University Press, Oxford, 34-38.

Fairbrother JE and Beaumont AR (1993). Heterozygote deficiencies in a cohort of newly settled Mytilus edulis spat. $J$. Mar. Biol. Assoc. UK. 73: 647-653.

Fischer D and Bachmann K (1998). Microsatellite enrichment in organisms with large genomes (Allium cepa L.). Biotechniques 24: 796-800, 802.

Gardner MG, Cooper SJB, Bull CM and Grant WN (1999). Isolation of microsatellite loci from a social lizard Egernia stokesii, using a modified enrichment procedure. J. Hered. 90: 301-304.

Geist J and Kuehn R (2005). Genetic diversity and differentiation of central European freshwater pearl mussel (Margaritifera margaritifera L.) populations: implications for conservation and management. Mol. Ecol. 14: 425-439.

Goudet J (1995). FSTAT (version 1.2): a computer program to calculate F-statistics. J. Hered. 86: 485-486.

Hamilton MB, Pincus EL, Di FA and Fleischer RC (1999). Universal linker and ligation procedures for construction of genomic DNA libraries enriched for microsatellites. Biotechniques 27: 500-507.

Hubert S and Hedgecock D (2004). Linkage maps of microsatellite DNA markers for the Pacific oyster Crassostrea gigas. Genetics 168: 351-362.

Jeong DS, Umino T, Kuroda K, Hayashi M, et al. (2003). Genetic divergence and population structure of black sea bream 
Acanthopagrus schlegeli inferred from microsatellite analysis. Fish. Sci. 69: 896-902.

Karagyozov L, Kalcheva ID and Chapman VM (1993). Construction of random small-insert genomic libraries highly enriched for simple sequence repeats. Nucleic Acids Res. 21: 3911-3912.

Levinson G and Gutman GA (1987). High frequencies of short frameshifts in poly-CA/TG tandem repeats borne by bacteriophage M13 in Escherichia coli K-12. Nucleic Acids Res. 15: 5323-5338.

Li Q and Kijima A (2002). Identification of novel microsatellite loci in the Pacific oyster (Crassostrea gigas) by magnetic bead hybridization selection. Tohoku. J. Agric. Res. 53: 25-32.

Li TW, Li CH, Song LS and Su XR (2003). RAPD variation within and among five populations of Tegillarca granosa. Biodivers. Sci. 11: 118-124.

Marshall TC, Slate J, Kruuk LE and Pemberton JM (1998). Statistical confidence for likelihood-based paternity inference in natural populations. Mol. Ecol. 7: 639-655.

Powell W, Gordon CM and Provan J (1996). Polymorphism revealed by simple sequence repeats. Trends. Plant. Sci. 1: 215-222.

Rassmann K, Schlotterer C and Tautz D (1991). Isolation of simple-sequence loci for use in polymerase chain reactionbased DNA fingerprinting. Electrophoresis 12: 113-118.

Raymond M and Rousset F (1995). Genepop (version 1.2): population genetics software for exact tests and ecumenicism. J. Hered. 86: 248-249.

Rosas KG, Perez-Buitrago N, Acevedo JP, Martinez N, et al. (2008). Development and characterization of 11 microsatellite loci for the Mona Island iguana (Cyclura cornuta stejnegeri). Mol. Ecol. Resour. 8: 825-827.

Schlötterer C and Tautz D (1992). Slippage synthesis of simple sequence DNA. Nucleic Acids Res. 20: 211-215.

Scott KD, Eggler P, Seaton G, Rossetto M, et al. (2000). Analysis of SSRs derived from grape ESTs. Theor. Appl. Genet. 100: 723-726.

Wang RX, Li TW, Lu ZM, Su XR, et al. (2005). Study on genetic variation by isozyme in different populations of bloody clam Tegillarca granosa. Oceanol. Etlimnol. Sin. 36: 227-234.

Wierdl M, Dominska M and Petes TD (1997). Microsatellite instability in yeast: dependence on the length of the microsatellite. Genetics 146: 769-779.

Wright S (1978). Evolution and the Genetics of Population. Variability Within and Among Natural Population. University of Chicago Press, Chicago.

Yu H and Li Q (2007). Genetic variation of wild and hatchery populations of the Pacific oyster Crassostrea gigas assessed by microsatellite markers. J. Genet. Genomics 34: 1114-1122.

Zhang YP, Lin ZH and Ying XP (2004). Morphological variations and discriminant analysis of different geographical populations of Tegillarca granosa. J. Fish. China 28: 339-342.

Zhao YY, Zhu XC, Sun XW and Liang L (2006). Microsatellite enrichment by magnetic beads in Japanese scallops (Patinopecten yessoensis). J. Fish. Sci. China 13: 749-755. 